\title{
Initial Antibiotic Selection of Patients Presenting with Infection after Transrectal Prostate Biopsy Leading to a Quality Improvement Initiative
}

\author{
Steven R. Madsen, Elizabeth Rourke, Stephen Overholser, Michael A. Liss \\ Department of Urology, University of Texas Health Science Center San Antonio South Texas Veterans Affairs Healthcare, \\ San Antonio, TX, USA \\ Email: stevenrmadsen@gmail.com
}

How to cite this paper: Madsen, S.R., Rourke, E., Overholser, S. and Liss, M.A. (2017) Initial Antibiotic Selection of Patients Presenting with Infection after Transrectal Prostate Biopsy Leading to a Quality Improvement Initiative. Open Journal of Urology, 7, 131-137.

https://doi.org/10.4236/oju.2017.78016

Received: July 27, 2017

Accepted: August 15, 2017

Published: August 18, 2017

Copyright $\odot 2017$ by authors and Scientific Research Publishing Inc. This work is licensed under the Creative Commons Attribution International License (CC BY 4.0).

http://creativecommons.org/licenses/by/4.0/

\begin{abstract}
Background: The incidence of infection associated with transrectal prostate biopsy has been increasing largely due to fluoroquinolone resistance (FQR). Purpose: To identify the antibiotic prescribing patterns employed when men seek medical professionals due to infectious complications of prostate biopsy, and employ a quality improvement initiative to improve antibiotic selection. Methods: A retrospective review determined the percentage of patients who presented to the emergency department (ED) after TRUS biopsy, and whether they were given appropriate antibiotic therapy. Prospective quality improvement was initiated by obtaining cultures via rectal swab at the time of transrectal biopsy in order to allow culture results and fluoroquinolone sensitivities to be available in the electronic medical record. The provider to guide antibiotic selection if the patient returned with infection symptoms could utilize the data. Findings: From 10/2009 to 6/2014, 0.5\% (9/1724) of patients who underwent TRUS prostate biopsy returned to the ED with infection. Seven patients had complete data and $4(0.57 \%)$ were given appropriate initial antibiotic treatment. To improve antibiotic selection, the 119 men who underwent rectal swab culture prior to biopsy $19.3 \%$ (23/119) showed FQR on rectal culture. $4 \%(5 / 119)$ were hospitalized with infection, demonstrating a significant increase in infection incidence from retrospective chart review $(0.5 \%$ to $4 \%, \mathrm{p}=$ $0.0013)$. Of these 5 men, $60 \%(3 / 5)$ had FQR on rectal culture. Conclusions: Rectal culture at the time of biopsy may lead to faster recognition of resistant bacteria when presented with prostate biopsy infection.
\end{abstract}

\section{Keywords}

Prostate Biopsy, Infection, Antibiotic Resistance, Fluoroquinolone 


\section{Introduction}

Transrectal ultrasound guided (TRUS) prostate biopsy is a widely used, minimally invasive procedure implemented in the initial patient work-up for prostate cancer [1]. During the procedure, a needle traverses both the anterior wall of the rectum and the prostate, potentially introducing colonic pathogens into the prostate and surrounding tissues. The bacteria found in the rectum at the time have been confirmed in the blood of post prostate biopsy septicemia confirming the bacteria directly come from the rectal flora [2]. Fluoroquinolone (FQ) antibiotics have historically been the standard prophylactic therapy used to prevent infection following biopsy. Though complications associated with this procedure are rare $(1 \%-6 \%)$, the incidence of post-biopsy infections has been increasing over the last decade [1] [3] [4] [5] [6]. Additionally, recent studies have demonstrated an increased incidence of FQ resistance in blood and urine cultures of patients presenting with infectious complications after TRUS prostate biopsy [6] [7] [8] [9]. Improvement can be made in the initial treatment of patients presenting with infections after prostate biopsy. Our purpose is to identify the antibiotic prescribing patterns employed when men seek medical professionals due to infectious complications of TRUS prostate biopsy. Due to the high resistance rate of trimethoprim/sulfamethoxazole and ciprofloxacin, these medications may not be appropriate to treat biopsy complications. We then seek to utilize a quality improvement initiative at the Audie L. Murphy Veterans Affairs Hospital (ALMVAH) to provide an alternative approach to guide antibiotic selection.

\section{Methods}

\subsection{Retrospective Review}

A retrospective chart review was performed for all TRUS prostate biopsies performed at ALMVAH within the last 5 years (10/2009 to 6/2014). All patients who presented to the Emergency Department (ED) shortly after biopsy were identified for further chart review. The patients who returned to the ED for a potentially biopsy-related infection were identified, and the antibiotics administered in the emergency department were recorded to identify the number of patients treated with FQ versus any other antibiotic therapy. A simple analysis was performed to identify the percentage of patients who presented to the emergency department with a biopsy related infection, and the percentage of those returning patients who were treated in the emergency department with appropriate antibiotic therapy.

\subsection{Quality Improvement}

In order to determine colonization and FQ resistance, we prospectively cultured patients undergoing TRUS biopsy with rectal swab prior to biopsy from July 6th, 2015 to October 1st, 2015. The Liquid Stuart Medium swab and transport system (Copan Diagnostics, Murrieta, CA) was performed immediately prior to placement of the ultrasound probe and sent to microbiology where it was inoculated 
onto MacConkey agar containing $10 \mu \mathrm{g} / \mathrm{ml}$ of ciprofloxacin (Hardy Diagnostics, Santa Maria, CA). As a control, the sample was also inoculated onto regular MacConkey agar to ensure that enteric bacteria were indeed on the swab. If after the 24-hour incubation there was no growth on the ciprofloxacin-infused MacConkey agar and there was growth of normal flora on the other agars, the rectal flora was assumed to be ciprofloxacin-sensitive. Conversely, any growth of Gramnegative (GN) rods on the ciprofloxacin-infused MacConkey agar was presumed to be ciprofloxacin-resistant. A representative of each distinct colony morphotype was then run through the Vitek 2 analyzer (BioMerieux, Durham, NC) for identification by GN cards and for sensitivity testing by Antibiotic Susceptibility Testing (AST) cards using Clinical and Laboratory Standards Institute Interpretative Criteria [10]. The result was then placed into the Computerized Patient Record System (CPRS) to be available for clinical providers to view the results and choose alternate antibiotics.

\section{Results}

\subsection{Retrospective Review}

Of the 1724 patients who underwent TRUS prostate biopsy at ALMVAH, 0.5\% (9) of patients presented to the Emergency department with a biopsy related infection. Of the 9 patients who presented with infection, $78 \%$ (7) were treated with antibiotics in the $\mathrm{ED}$, one patient received no antibiotic treatment until admission to a medicine team, and one patient was treated at an outside hospital. Of those patients treated in the ALMVAH ED, 57\% (4 of 7) were given appropriate antibiotic treatment (Figure 1).

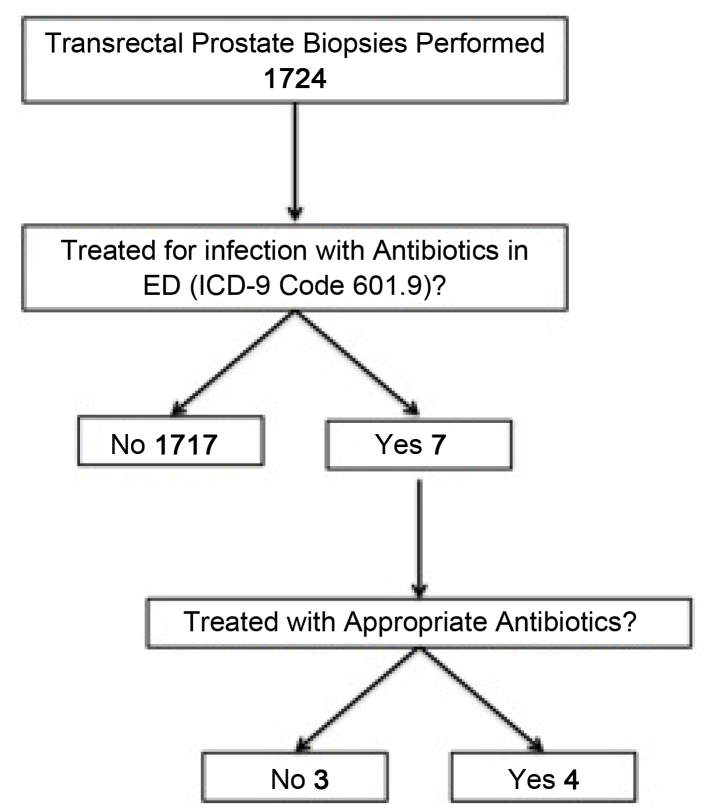

Figure 1. Flow diagram using ICD9 diagnosis codes for prostate biopsy infections at the south Texas veterans affairs medical center from 10/2009 to 6/2014. 
Of the patients given inappropriate antibiotic treatment in the ED, 2 were given trimethoprim-sulfamethoxazole, and one was given ciprofloxacin (Table 1).

\subsection{Quality Improvement}

From July $6^{\text {th }} 2015$ until January 1st, 2016 we retrospectively reviewed charts of men who underwent prostate biopsy at the South Texas Audie Murphy Veterans Hospital and received a rectal culture at the same time. We documented their antibiotic use, rectal culture result at biopsy, and infection rate. We identified 119 men of which 33 (28\%) received an $80 \mathrm{mg}$ of gentamicin in addition to a FQ for prophylaxis. The rectal culture results showed a $19.3 \%(23 / 119)$ FQR and 5 patients $(4 \%)$ were hospitalized with infection. This is a significant increase in infections complications $(0.5 \%$ to $4 \%, \mathrm{p}=0.0013)$. Sixty percent $(3 / 5)$ of patients hospitalized for post prostate biopsy infection had FQR E. coli confirmed with blood and/or urine cultures (Table 2).

Table 1. Initial antibiotic selection in men presenting with infection symptoms after transrectal prostate biopsy.

\begin{tabular}{ccc}
\hline Patient & Antibiotics Given & Retrospective Appropriate treatment $^{*}$ \\
\hline 1 & Ceftriaxone & Yes \\
2 & Ceftriaxone & Yes \\
3 & Trim-Sulfa & No \\
4 & Ciprofloxacin & No \\
5 & Trimethoprim-Sulfamethoxazole & No \\
6 & Ceftriaxone & Yes \\
7 & Zosyn + Gentamycin & Yes \\
\hline
\end{tabular}

*Appropriate treatment is based on the resistance profile of E. coli isolated from the rectum at the time of prostate biopsy at our institution. If the overall resistance level to a particular antibiotic is $>20 \%$, this antibiotic may not be considered an appropriate first choice. The providers did not have access to culture data as this data was collected by ICD9 diagnosis codes, while the rectal cultures were started after this data was obtained. Therefore, this table represents the possibility to improve antibiotic prescribing habits IF culture data would have been known.

Table 2. Culture and sensitivities for TRUS biopsy related infection requiring hospitalization. Amp = Ampicillin; A/S = Ampicillin/Sulbactam; Cipro = Ciprofloxacin; T/S = Trimethoprim/Sulfamethoxazole; Gent $=$ Gentamicin.

\begin{tabular}{ccccc}
$\begin{array}{c}\text { Trus } \\
\text { Infection }\end{array}$ & $\begin{array}{c}\text { Rectal } \\
\text { Culture }\end{array}$ & $\begin{array}{c}\text { Rectal Culture } \\
\text { Resistance }\end{array}$ & $\begin{array}{c}\text { Blood culture } \\
\text { Resistance }\end{array}$ & $\begin{array}{c}\text { Urine Culture } \\
\text { resistance }\end{array}$ \\
\hline 1 & Incomplete & N/A & Amp, T/S, A/S, Cipro Amp, T/S, A/S, Cipro & Amp, \\
2 & FQR E. coli & Amp, T/S, Gent, Cipro & Amp, T/S, Gent, Cipro & No Growth \\
3 & FQR E. coli & Cipro only & $\begin{array}{c}\text { No resistant } \\
\text { antibiotics }\end{array}$ & Cipro Only \\
4 & $\begin{array}{c}\text { No FQR } \\
\text { organism }\end{array}$ & N/A & $\begin{array}{c}\text { No resistant } \\
\text { Antibiotics }\end{array}$ & No Growth \\
5 & $\begin{array}{c}\text { No FQR } \\
\text { organism }\end{array}$ & N/A & No growth & $\begin{array}{c}\text { No Resistant } \\
\text { Antibiotics }\end{array}$ \\
\hline
\end{tabular}


Patient one had insufficient specimen for speciation of rectal culture and was hospitalized twice for insufficient treatment of FQR E. coli. Patient two and three had a positive FQR $E$. coli rectal swab with complete sensitivities available within 48 hours of hospitalization allowing for prompt transition from IV to oral antibiotics and decreased length of stay compared to patient one, who remained hospitalized until blood and urine culture sensitivities were completed. Patients four and five had negative rectal cultures but a positive urine or blood culture for E. coli and were transitioned to oral antibiotics hospital day six and three respectively.

\section{Discussion}

Infection associated with TRUS prostate biopsy is an uncommon presentation to most Emergency Departments; however increased from historical 0.5\% to nearly $4 \%$ in the 6 months of our study $(\mathrm{p}=0.0013)$. Therefore, providers in emergency departments may not be fully aware of the current increase of infections complications from fluoroquinolone resistance E. coli that has caused the majority of infections. Trimethoprim sulfamethoxazole or another fluoroquinolones would not be appropriate antibiotic selections for men presenting with infectious complications from prostate biopsy.

Our examination of the antibiotic resistance profiles of the 21 patients that were colonized with fluoroquinolone resistant E. coli (Figure 2) show a similar resistance profile as published in other studies [11].

Attention is brought to gentamicin because $28 \%$ of men in our study were deemed "at risk" for infection and were given this antibiotic to prevent infection. Two specific issues relating to gentamicin are important to discuss including weight based dosing and secondary resistance. First, gentamicin should be dosed by the patient's weight. The American Urologic Association Best Practice Policy Statement on Urologic Surgery Antimicrobial Prophylaxis recommends $5 \mathrm{mg} / \mathrm{kg}$ dosing [12]. After discussion with our pharmacy department, they feel that 2 $\mathrm{mg} / \mathrm{kg}$ dosing was sufficient for prostate biopsy prophylaxis. The vast majority of our patients are over $80 \mathrm{~kg}$, which means at least a dose of $160 \mathrm{mg}$ would be needed for sufficient antimicrobial coverage. Second, 38\% of the 21 fluoroquinolone

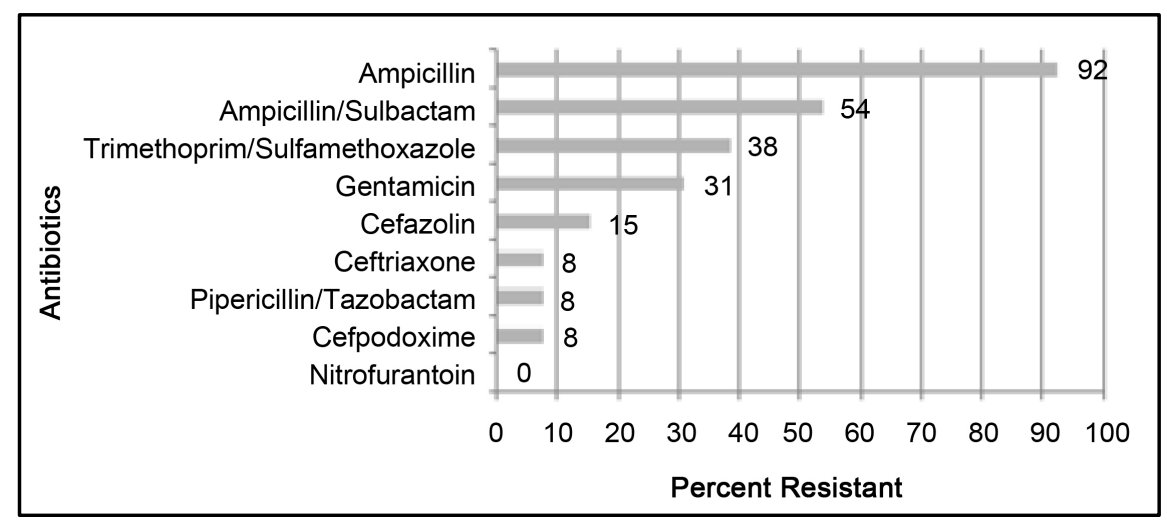

Figure 2. FQR E. coli rectal swab resistance profile. $\mathrm{N}=24$. 
resistant isolates present in the rectum at the time of prostate biopsy were also resistant to gentamicin. Therefore, a cephalosporin would be a better choice for augmented prophylaxis regimens or the culture should be done prior to the biopsy to provide culture-directed antibiotics prophylaxis.

The purpose of the study was to improve antibiotic selection upon presentation for transrectal prostate biopsy infection. Our 5-year retrospective review of TRUS biopsies at the ALMVAH demonstrated only 9 cases of biopsy related infection ending in June of 2014. All patients were initially evaluated in the emergency department, however 3 patients either received inappropriate antibiotic treatment, or appropriate antibiotic treatment was delayed until after Urology had been consulted. The overall low incidence of TRUS biopsy related infections might contribute to the delay in appropriate antibiotic selection by emergency department providers or primary care physicians. The impetus is on the urologist to educate the patient to not only seek prompt treatment for febrile illness or suspected infection after biopsy but to remind the patient to bring the antibiotic bottle with them to the emergency room. We also could perform a rectal culture at the time of biopsy in order to expedite the process of correct antibiotic selection if the patient returns to the emergency department.

Therefore, to improve antibiotic selection a rectal swab culture was taken at the time of biopsy to guide providers in the selection of appropriate antibiotic treatment for patients with biopsy related infections. We performed rectal cultures on 119 men and when the culture was available, the emergency room providers were able to view the results in the electronic medical record system. This essentially eliminated inappropriate antibiotic selection and allowed from prompt treatment of antibiotics.

Strengths of the study include the retrospective large cohort at the diverse Veterans Affairs medical center in San Antonio Texas. The ideal scenario would be to perform the rectal culture 2 weeks prior to the prostate biopsy in order to change prophylaxis. Unfortunately, many centers are unable to accomplish this goal due to various logistics including patient, physician, laboratory, and expertise factors. The technique of applying an opportunity for improvement in the selection of antibiotics if a patient returns due to infectious complications could not only provide initial experience in the rectal culture technique for physicians and their respective laboratories, but provide valuable information to providers for antibiotic selection. Weaknesses of the study include the small number of patients with infection and inability to provide multivariable analysis on other causes of infection due to low numbers. On average infection only happens in $1 \%-3 \%$ of the prostate biopsy population.

\section{Conclusion}

Our study shows a very significant rise in prostate biopsy infection. Historically, these infections may not have been treated appropriately due to the rare occurrence and lack of awareness of this infectious problem unique to the specific pa- 
tient population. We believe that the introduction of rectal cultures prior to prostate biopsy or at the time of biopsy, along with education of emergency department providers regarding the high level of FQ resistance in TRUS prostate biopsy patients may lead to improved initial antibiotic selection, reduced use of broad spectrum antibiotics, and decreased hospital stay. Further investigation of the potential benefits of rectal swab culture taken at the time of biopsy can aid in determining the possible benefits of rectal swab culture in patient care.

\section{References}

[1] Loeb, S., Carter, H.B., Berndt, S.I., et al. (2011) Complications after Prostate Biopsy: Data from SEER-Medicare. Journal of Urology, 186, 1830.

[2] Liss, M.A., Johnson, J.R., Porter, S.B., et al. (2014) Clinical and Microbiological Determinants of Infection after Transrectal Prostate Biopsy. Clinical Infectious Diseases, 60, 979-987.

[3] Dumford, D., Suwantarat, N., Bhasker, V., et al. (2013) Outbreak of Fluoroquinolone-Resistant Escherichia coli Infections after Transrectal Ultrasound-Guided Biopsy of the Prostate. Infection Control \& Hospital Epidemiology, 34, 269. https://doi.org/10.1086/669512

[4] Mosharafa, A.A., Torky, M.H., El Said, W.M., et al. (2011) Rising Incidence of Acute Prostatitis Following Prostate Biopsy: Fluoroquinolone Resistance and Exposure Is a Significant Risk Factor. Urology, 78, 511.

[5] Nam, R.K., Saskin, R., Lee, Y., et al. (2010) Increasing Hospital Admission Rates for Urological Complications after Transrectal Ultrasound Guided Prostate Biopsy. Journal of Urology, 183, 963.

[6] Wagenlehner, F.M., van Oostrum, E., Tenke, P., et al. (2013) Infective Complications after Prostate Biopsy: Outcome of the Global Prevalence Study of Infections in Urology (GPIU) 2010 and 2011: A Prospective Multinational Multicentre Prostate Biopsy Study. European Urology, 63, 521.

[7] Liss, M.A., Taylor, S.A., Batura, D., et al. (2014) Fluoroquinolone Resistant Rectal Colonization Predicts Risk of Infectious Complications after Transrectal Prostate Biopsy. Journal of Urology, 192, 1673.

[8] TukenmezTigen, E., Tandogdu, Z., Ergonul, O., et al. (2014) Outcomes of Fecal Carriage of Extended-Spectrum Beta-Lactamase after Transrectal UltrasoundGuided Biopsy of the Prostate. Urology, 84, 1008.

[9] Williamson, D.A., Barrett, L.K., Rogers, B.A., et al. (2013) Infectious Complications Following Transrectal Ultrasound-Guided Prostate Biopsy: New Challenges in the Era of Multidrug-Resistant Escherichia coli. Clinical Infectious Diseases, 57, 267. https://doi.org/10.1093/cid/cit193

[10] Clinical and Laboratory Standards Institute (2010) Performance Standards for Antimicrobial Susceptibility Testing: 20th Informational Supplement M100-S20. Clinical and Laboratory Standards Institute, NCCLS, Wayne.

[11] Liss, M.A., Peeples, A.N. and Peterson, E.M. (2011) Detection of FluoroquinoloneResistant Organisms from Rectal Swabs by Use of Selective Media Prior to a Transrectal Prostate Biopsy. Journal of Clinical Microbiology, 49, 1116. https://doi.org/10.1128/JCM.01885-10

[12] Wolf, J.S., Bennett, C.J., Dmochowski, R.R., et al. (2008) Best Practice Policy Statement on Urologic Surgery Antimicrobial Prophylaxis. Journal of Urology, 179, 1379. 
Submit or recommend next manuscript to SCIRP and we will provide best service for you:

Accepting pre-submission inquiries through Email, Facebook, LinkedIn, Twitter, etc. A wide selection of journals (inclusive of 9 subjects, more than 200 journals)

Providing 24-hour high-quality service

User-friendly online submission system

Fair and swift peer-review system

Efficient typesetting and proofreading procedure

Display of the result of downloads and visits, as well as the number of cited articles Maximum dissemination of your research work

Submit your manuscript at: http://papersubmission.scirp.org/

Or contact oju@scirp.org 